\title{
Extradural Chondroma on the Lumbar Spine: A Case Report
}

\author{
Yong Guk Kim¹, Tae Wan Kim¹, Eun Ju Kim², Kwan Ho Park \\ Departments of ${ }^{1}$ Neurosurgery, ${ }^{2}$ Pathology, VHS Medical Center, Seoul, Republic of Korea
}

Corresponding author: Tae Wan Kim

Department of Neurosurgery, VHS Medical

Center, 53, Jinhwangdo-ro 61-gil, Gangdong-gu,

Seoul 05368, Republic of Korea

Tel: $+82-2-2225-1363$

Fax: $+82-2-2225-4152$

E-mail: euro3399@naver.com

Received: May 28, 2020

Revised: June 11, 2020

Accepted: June 24, 2020
Chondromas are benign tumors originating from cartilaginous structures. Chondromas located on the spine are rare. We report the case of a 74-year-old woman who complained of back pain and right lower extremity radiating pain. Magnetic resonance imaging revealed an oval-shaped mass-like lesion in the epidural space at the right foraminal zone on L2-3. The mass had intermediate signal intensity on T1- and T2- weighted images. She underwent surgical tumor removal and the symptoms gradually improved. The mass lesion was confirmed by histopathological examination as a chondroma. We report a rare case of lumbar spinal chondroma accompanied by radicular symptoms.

Key Words: Chondroma; General surgery; Spine

\section{INTRODUCTION}

Chondromas are benign tumors arising from cartilaginous structures and are susceptible to metaplasia and malignant degeneration $^{8)}$. Most chondromas are occurring in the fingers, followed by the hands, toes, and feet. Chondromas originating in the spine are very rare.

The common site of the spine is not clear. But, the previously reported cases of spinal chondromas in cervical and lumbar lesions are more than 15 cases. Relatively, only 1 case is reported in the thoracic spine ${ }^{6)}$. Most spinal chondromas remain asymptomatic. But, few patients have paravertebral swelling and pain, and some complain of radicular pain and neurologic deficits.

We report a rare case of an extradural lumbar spinal chondroma located in the foraminal zone resulting in radicular pain.

\section{CASE REPORT}

A 74-year-old woman presented at our department complaining of back pain and right lower extremity pain and numbness for 1 year. She underwent lumbar surgery at the L4-5 level at another hospital.

On neurologic examination, motor weakness, muscle atrophy, and pathologic reflex were not noted.

Magnetic resonance imaging (MRI) demonstrated an ovalshaped mass-like lesion in the epidural space at the right foraminal zone on L2-3. The mass had an isointense signal on T1- and T2-weighted images (WIs). The mass showed strong peripheral rim-like enhancement and central non-enhancing foci (Fig. 1).

She underwent surgery. After hemilaminectomy and facetectomy, total tumor removal was performed. We conducted
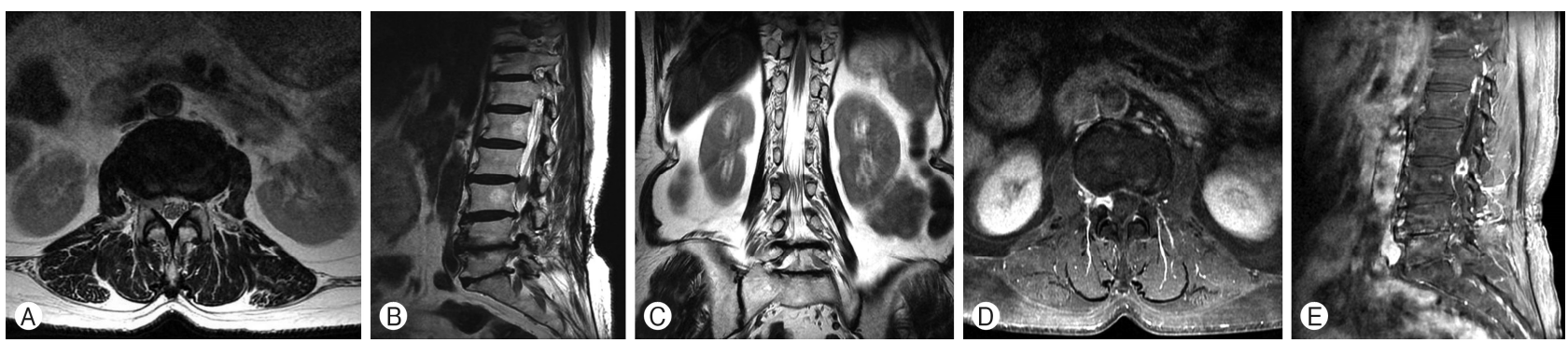

Fig. 1. Magnetic resonance images showing an oval-shaped mass in the foraminal zone. (A) T2-weighted axial, (B) T2-weighted sagittal, (C) T2-weighted coronal images. (D, E) After enhancement, well-demarcated rim enhancement was observed. 
transpedicular screw fixation for the prevention of postoperative spinal instability. Decompression of the dura mater was observed intraoperatively. The mass was pinkish, soft, friable, and well-dissected from the dura (Fig. 2).

After surgical treatment, back pain and radiating pain were

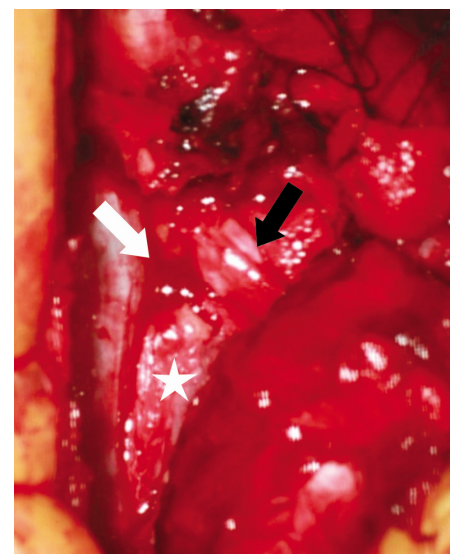

Fig. 2. After facetectomy, a well-capsulated pinkish elongated mass was noted (star) between the nerve root (black arrow) and lateral margin of the dura (white arrow).

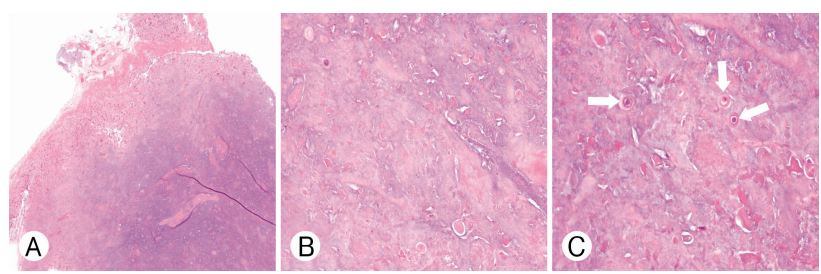

Fig. 3. (A) Histopathological examination revealed a mass-like lesion with a thin fibrous capsule and chondroid matrix (hematoxylin and eosin stain [H\&E], $\times 40$ magnification), which a hypocellular lesion with an abundant amount of hyaline cartilage matrix. (B) Chondrocytes are well-suited within lacunar spaces $(\mathrm{H} \& \mathrm{E}, \times 200$ magnification). (C) Chondrocytes (white arrows) have finely granular eosinophilic cytoplasm. The nuclei are typically small and round with condensed chromatin (H\&E, $\times 400$ magnification).

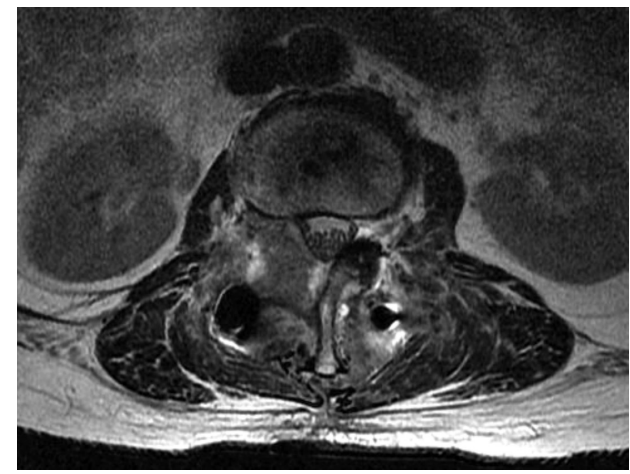

Fig. 4. Follow-up magnetic resonance image showing complete removal of the mass. relieved. Histopathological examination confirmed the mass to be a chondroma (Fig. 3).

On a follow-up MRI examination, total mass removal was verified (Fig. 4). The patient was discharged without complications.

\section{DISCUSSION}

Tumors originating from the cartilage are classified as chondromas, osteochondromas, chondroblastomas, and chondromyxoid fibromas. Chondromas are subdivided into 2 types: enchondroma and periosteal chondroma ${ }^{7}$. When chondromas are found distant from the bones, they are referred to as soft tissue chondromas ${ }^{11)}$. Enchondromas tend to grow as expansile patterns, and periosteal chondromas are exophytic ${ }^{9)}$. Spinal chondromas may be derived from hyperplasia of immature spinal cartilage from metaplasia of the connective tissue in contact with the spine or annulus fibrosus ${ }^{8)}$.

Chondromas in the spine constitute about 3\% and $4 \%$ of all chondromas ${ }^{4,7,9)}$. Cartilage-forming tumors account for $2 \%$ of all spinal tumors ${ }^{3}$. Chondromas are involved in the vertebral structure, vertebral body, neural arch, and spinous and transverse processes. The neural arch is the most common site of lumbar spine chondromas ${ }^{7}$. A rare spinal intradural chondroma has also been reported ${ }^{4)}$.

Most cervical spine chondromas occur in young male adults, and the initial symptoms are motor weakness and sensory change $^{1)}$. Neurologic symptoms are more common in adults aged $<40$ years ${ }^{5}$. Symptomatic chondromas on the lumbar spine are rare and can result in back pain, numbness, and hypoesthesia of lower extremities ${ }^{2)}$, but radicular symptoms are not common ${ }^{3)}$. A large number of chondromas on the lumbar spine are asymptomatic because of their slow-growing nature. When those are not involving the spinal canal, most of the cases are also asymptomatic.

A differential diagnosis between benign chondroma and welldifferentiated chondrosarcoma is difficult, but important ${ }^{2}$. Chondromas are usually isolated as single lesions and can undergo malignant transformation. When multiple chondromas are noted, Ollier disease and Maffucci syndrome should be considered. Multiple chondromas can undergo more sarcomatous changes than a single lesion".

Plain radiography may show only smooth erosion of the bony structure, which could have been in direct contact with tumors or may also show a well-circumscribed lytic lesion with a widened neural foramen. Computed tomography (CT) and MRI are important tools for evaluating tumor growth, invasion, and the relation of adjacent structures ${ }^{3)}$. CT can be used to demonstrate hyperdense calcified lesion ${ }^{9,12)}$, and is the preferred modality to demonstrate osseous structures of tumors. Furthermore, extraosseous soft-tissue edema close to the mass can be detected on T2-WIs". The relatively small size and absence of soft tissue invasion suggest a benign chondroma 
rather than a malignant nature ${ }^{6}$. Chondromas usually present as an isointense signal on T1-WIs and hypointense to isointense signals on T2-WIs, but the signals can be variable. Peripheral rim enhancement has been observed on gadolinium-enhanced MRI scans ${ }^{7,10,12)}$.

Chemotherapy is ineffective, and radiotherapy is unnecessary if total tumor removal is performed. Radiotherapy is considered when the tumor is not completely removed ${ }^{1)}$.

Asymptomatic chondromas in the spinal canal are more common than symptomatic chondromas in the spine. We report a rare case of lumbar spinal chondroma accompanied by radicular symptoms.

\section{CONCLUSION}

Though spinal chondromas are very rare, extradural spinal tumors should still be considered in a differential diagnosis. Complete surgical removal is recommended for pathological confirmation and prevention of recurrence and malignant transformation. Long-term follow-up may be needed.

\section{CONFLICTS OF INTEREST}

No potential conflict of interest relevant to this article was reported.

\section{REFERENCES}

1. Byun YH, Sohn S, Park SH, Chung CK: Cervical spine chondroma compressing spinal cord: A case report and literature review.
Korean J Spine 12:275-278, 2015

2. Cetinkal A, Güven G, Topuz AK, Colak A, Demircan MN, Haholu A: Lumbar spinal chondroma presenting with radiculopathy: Case report. Turk Neurosurg 18:397-399, 2008

3. Gaetani P, Tancioni F, Merlo P, Villani L, Spanu G, Baena RR: Spinal chondroma of the lumbar tract: case report. Surg Neurol 46:534-539, 1996

4. Hori Y, Seki M, Tsujio T, Hoshino M, Mandai K, Nakamura $\mathrm{H}$ : Intradural chondroma in the cervical spine: Case report. J Neurosurg Spine 26:257-259, 2017

5. Inoue T, Ohara Y, Niiro T, Endo T, Tominaga T, Mizuno JI: Cervical periosteal chondroma causing spinal cord or nerve compression: 2 case reports and literature review. World Neurosurg 114:99-105, 2018

6. Kang DH, Kang BS, Sim HB, Kim M, Kwon WJ: Periosteal chondroma with spinal cord compression in the thoracic spinal canal: A case report. Skeletal Radiol 45:1133-1137, 2016

7. Kim DH, Nam KH, Choi BK, Han I: Lumbar spinal chondroma presenting with acute sciatica. Korean J Spine 10:252-254, 2013

8. Lozes G, Fawaz A, Perper H, Devos P, Benoit P, Krivosic I, et al.: Chondroma of the cervical spine. Case report. J Neurosurg 66:128-130, 1987

9. McLoughlin GS, Sciubba DM, Wolinsky JP: Chondroma/Chondrosarcoma of the spine. Neurosurg Clin N Am 19:57-63, 2008

10. Ogata T, Miyazaki T, Morino T, Nose M, Yamamoto H: A periosteal chondroma in the lumbar spinal canal. Case report. J Neurosurg Spine 7:454-458, 2007

11. Pace J, Lozen AM, Wang MC, Cochran EJ: Extradural chondroma presenting as lumbar mass with compressive neuropathy. J Craniovertebr Junction Spine 5:131-133, 2014

12. Russo V, Platania N, Graziano F, Albanese V: Cervical spine chondroma arising from C5 right hemilamina: A rare cause of spinal cord compression. Case report and review of the literature. J Neurosurg Sci 54:113-117, 2010 